\title{
When is recall higher than recognition?
}

ENDEL TULVING

UNIVERSITY OF TORONTO

Sixteen Ss learned a list of 48 paired words (A-B pairs) to a criterion of two perfect trials and were then tested for the recognition of B-members of all pairs. Approximately $10 \%$ of the learned words were not correctly identified in the recognition test. These data show that recall is higher than recognition when retrieval cues present at the recall test are more effective in providing access to stored information than are retrieval cues present at the recognition test.

Recognition tests of memory usually yield higher scores of retention than do recall tests. Such superiority of recognition over recall is explained in terms of two factors: (1) the differences in the number of alternatives from among which correct responses are to be selected (Brown, 1965; Davis, Sutherland, \& Judd, 1961; Slamecka, 1967), and (2) the differences in the amount and nature of retained information necessary for the identification and for the unaided reproduction of learned items (McNulty, 1965). If the two factors are equated in recall and in recognition, then the two measures should, and sometimes do, yield identical estimates of retention.

No extant theoretical formulation of recall and recognition specifies any conditions under which recall might be superior to recognition, even though some experiments demonstrating this relationship have been reported (e.g., Bahrick \& Bahrick, 1964; Lachman \& Field, 1965). In these experiments, however, recognition tests of individual items were paced by $E$, and S's failure to correctly identify items learned earlier may have been related to lack of time for considering the alternatives available to $S$ on the basis of unaided recall. The present paper reports a simple experiment showing that Ss sometimes fail to recognize items they can reproduce even if they have unlimited time in the recognition test. An explanation of this phenomenon is also proposed. Method

Sixteen Ss, undergraduate and graduate psychology students of both sexes, participated in the experiment. Each $S$ learned a single list of 48 paired words (A-B pairs), under the typical paired-associate anticipation conditions, and was then tested for the recognition of $B$ members of the pairs.

Two different lists were used, each with eight Ss. In both lists, $A$ and $B$ members of all pairs were common monosyllabic English words. To eliminate the massive amount of experimental practice necessary for the mastery of a 48-pair list, A-B pairs were made up of words meaningfully related to each other. In List 1, for instance, pairs such as the following were used: TOOTH-ACHE, AIR-PORT, FLOOR-
SHOW, HOME-STEAD. The A members of pairs in List 2 were identical with the A members of List 1, but $B$ members were all different. Thus, the pairs in List 2 corresponding to the examples given from List 1 were: TOOTH-PICK, AIR-CRAFT, FLOORCLOTH, HOME-SICK.

Each $S$ was tested individually. On the first trial the prompting method was used. Each pair of words, hand-printed on a $3 \times 5$ card, was shown to $S$, followed by a card bearing only the $A$ member of the pair. The $S$ was asked to call out the $B$ member of the pair when presented with the $A$ member. No $S$ made any errors on this trial. Beginning with the second trial, the standard paired-associate method was used. A given $\mathrm{A}$ member was shown, the $\mathrm{S}$ asked to name the $B$ member and then, regardless of the $S^{\prime} s$ response, both $A$ and $B$ members of the same pair were presented. One trial consisted of the test and presentation of all 48 pairs. The presentation of cards bearing $A$ members and pairs was paced by $S$ and thus the rate varied from $S$ to $S$. On the average, Ss took approximately $5 \mathrm{~min}$ to go through the list on the second trial (first anticipation trial), but they speeded up in the course of practice, and on the fifth trial the average time per trial had been reduced to approximately $3 \mathrm{~min}$. Two different orders of presentation of pairs were used with each $S$, but these orders were different for different Ss.

Paired-associate training was continued with each $S$ until the $S$ had anticipated all 48 B members correctly on two consecutive trials. The $S$ was then given a sheet of paper on which were printed, in alphabetical order, the $96 \mathrm{~B}$ members of Lists 1 and 2 , and he was asked to check off all the words he had just learned. Thus, all 16 Ss took the same recognition test, with one-half of the items "old" for one group and the other half old for the other group. This procedure eliminated any possible effects of response bias or guessing on the recognition scores. Unlimited time was allowed for this test.

\section{Resul ts}

The data were pooled over both groups (1.e., both lists), since there were no apparent differences in the results of the two groups. The number of trials required to reach the criterion (excluding the first prompting trial, but including the two criterion trials) ranged from 5 to 9 , the mean for 16 Ss being 7.2. Every $S$ reached the criterion of two perfect trials immediately following his first perfect trial. It is a safe conclusion, therefore, that had the Ss been given another paced paired-associate test trial, their performance would have been perfect on that trial. 
The numbers of correct identifications of old items as old ranged from 36 to 47 , with a mean of 43.4 . Thus, on the average, Ss failed to recognize 4.6 items among the 48 that they had been able to recall in the presence of A words. The mean number of incorrect identifications of new items as old was .87 , and the median was zero.

\section{Discussion}

The results of this experiment clearly show that it is possible for Ss to recall-that is, to reproduce from memory-learned verbal units even if they cannot identify these units as old items in a recognition test. Thus, under the conditions of the present experiment, recall is superior to recognition. While the generality of these results-with respect to factors such as the nature of the material, length of the list, amount of practice given prior to the recognition and recall tests, and the like-remains to be determined, some relevant features of the conditions of the experiment must be identified for the purpose of the interpretation of the results.

The recall test was one involving aided or cued recall. The Ss reproduced each $B$ item in the presence of a specific retrieval cue, the corresponding $A$ item. Noncued recall of $B$ items certainly would have been considerably lower than the obtained cued recall (cf., Tulving \& Pearlstone, 1965), and also lower than the observed recognition performance. On the other hand, it is an equally safe conclusion that aided or cued recognition-recognition of a $B$ item in the presence of its corresponding $A$ item-would have been at least as high as cued recall. Recall cannot be higher than recognition as long as retrieval cues are identical, but it can be higher if retrieval cues are different in the two test situations.

The results of the present experiment suggest that in some cases the $A$ item was a more effective retrieval cue in providing access to the stored information about the related $B$ item than was the $B$ item in providing access to the stored information about its own "copy." This apparent paradox is resolved if we draw a distinction between nominal and functional memory units (Tulving, in press). Although the stored B item is nominally identical with the B item in the recognition test, the two need not represent identical subjective units. For instance, CRAFT in the recognition test may be coded differently from CRAFT in the higher-order unit of AIRCRAFT in the input list and may thus fail to provide access to desired information available in the memory store. Effectiveness of retrieval cues is critically dependent upon the extent to which they coincide with additional information stored about each to-be-remembered item at the time of input (Tulving \& Osler, in press). It is thus not surprising that some other item can be a more efficient cue for the retrieval of a given item than that item itself.

An important implication of the present results has to do with claims sometimes made that recognition "does not require retrieval" (Schonfield \& Robertson, 1966) or that "recognition eliminates the search or retrieval problem" (Murdock, in press). If recognition performance depends not only on the amount and organization of information in the memory store, but also on the number and nature of retrieval cues present at the time of the recognition test-as the present data clearly indicate it does-then it becomes as necessary for students of memory to worry about retrieval problems in recognition tests as it is necessary in situations when memory is tested with different kinds of recall tests. Consideration of the role of retrieval cues in both recall and recognition does at least make it possible to provide one answer to the question: "When is recall higher than recognition?" Recall is higher than recognition whenever retrieval cues present at the recall test are more effective in providing access to stored information than are retrieval cues present at the recognition test.

\section{References}

BAHRICK, H. P. \& BAHRICK, P. O. A re-examination of the interrelations among measures of retention. Quart. J. exp. Psychol., $1964,16,318-324$.

BROWN, J. A comparison of recognition and recall by a multipleresponse method. J. verbal Learn. verbal Behav., 1965, 4, 401-408.

DAVIS, R., SUTHERLAND, N. S., \& JUDD, B. R. Information content in recognition and recall. $J$. exp. Psychol, 1961, 61, 422-429.

LACHMAN, R., \& FIELD, W. H. Recognition and recall of verbal material as a function of degree of training. Psychon. Sci., 1965, 2, 225226.

McNULTY, J. A. An analysis of recall and recognition processes in verbal learning. J. verbal Learn. verbal Behav., 1965, 4, 430-436.

MURDOCK, B. B., Jr. Modality effects in short-term memory: Storage or retrieval? J. exp. Psychol, in press.

SLAMECKA, N. J. Recall and recognition in list-discrimination tasks as a function of the number of alternatives. J. exp. Psychol., 1967, 74, 187-192.

SCHONFIELD, D., \& ROBERTSON, B. Memory storage and aging. Canad. J. Psychol., 1966, 20, 228-236.

TULVING, E. Theoretical issues in free recall. In T. R. Dixon and D. L. Horton (Eds.) Verbal behavior and general behavior theory. Englewood Cliffs, N. J.: Prentice-Hall, in press.

TULVING, E., \& OSLER, S. Effectiveness of retrieval cues in memory for words. J. exp. Psychol, in press.

TULVING, E., \& PEARLSTONE, Z. Availability versus accessibility of information in memory for words. J. verbal Learn. verbal Behav., $1966,5,381-391$.

Note

1. This research was supported by the National Research Council Grant APB-39 and the National Science Foundation Grant GB 3710. The author is indebted to Miss Laurain King for collecting the data. 